\title{
Caracterização espectral de propriedades ópticas de aerossóis em região de floresta tropical
}

\author{
Jorge Almeida de MENEZES ${ }^{2 *}$, Rafael da Silva PALÁCIOS ${ }^{1}$, Evanízio Marinho de MENEZES JUNIOR², \\ Amazonino SOARES JÚNIOR ${ }^{2}$, José de Souza NOGUEIRA ${ }^{1}$
}

\author{
${ }^{1}$ Programa de Pós-Graduação em Física Ambiental, Universidade Federal de Mato Grosso, Cuiabá, MT, Brasil \\ ${ }^{2}$ Instituto de Educação, Agricultura e Ambiente, Universidade Federal do Amazonas, Humaitá, AM, Brasil. \\ *E-mail: jorgejr@ufam.edu.br
}

\begin{abstract}
Recebido em outubro/2017; Aceito em junho/2018.
RESUMO: Aerossóis atmosféricos desempenham um papel importante no equilíbrio de energia do sistema Terra-atmosfera. Medidas de aerossóis foram realizadas em área de floresta tropical utilizando fotômetro solar da rede global AERONET. Dados de 4 anos permitiram classificar as propriedades óticas dos aerossóis, definindo a climatologia para área de floresta tropical. Neste trabalho a profundidade óptica de aerossol (AOD) e expoente Ångström, $\alpha(440-870)$, foram utilizados para a análise espectral de aerossol. Médias de AOD(440nm) de 0,22 $( \pm 0,40)$, com média correspondente de $\alpha 1,27( \pm 0,39)$. A AOD $(440 \mathrm{~nm})$ mostra picos distintos para estação seca e chuvosa, sendo que podem estar relacionados com padrões sazonais de aerossóis característicos dessas regiões. $\mathrm{O} \alpha(440-870)$ apresentou modos de frequência que se relacionam com os principais tipos de aerossóis presentes em regiões de Floresta. Um cenário principal de aerossóis foi definido como aerossóis oriundos de queima de biomassa, com forte influência de fontes locais de aerossóis poluídos. Mistura de aerossóis estão presentes, enquanto que poeira em suspensão tem uma ocorrência pouco relevante.
\end{abstract}

Palavras-chave: AOD, expoente Ångström, climatologia.

\section{Spectral characterization of optical properties of aerosols in forest region}

\begin{abstract}
Atmospheric aerosols play an important role in the energy balance of the Earth-atmosphere system. Aerosol measurements were performed in rainforest area, Manaus_Embrapa site using solar photometer from the AERONET global network. Data from 4 years allowed to classify the optical properties of the aerosols, defining the climatology for the site. In this work, the aerosol optical depth (AOD) and Angström exponent, $\alpha$ (440-870), were used for aerosol spectral analysis. Mean AOD (440nm) of 0,22 ( \pm 0.40$)$, with corresponding mean of $\alpha 1,27( \pm 0.39)$. AOD $(440 \mathrm{~nm})$ shows distinct peaks for dry and rainy season, and may be related to seasonal aerosol patterns characteristic of these regions. The $\alpha$ (440-870) presented frequency modes that relate to the main types of aerosols present in Forest regions. A major aerosol scenario was defined as biomass-based aerosols, with strong influence from local sources of polluted aerosols. Mixtures of aerosols are present, while suspended dust has a slightly relevant occurrence.
\end{abstract}

Keywords: AOD, Ångström exponent, climatology.

\section{INTRODUÇÃO}

Aerossóis, partículas em suspensão no ar, com diversos atributos físicos e químicos, agem como um fator crucial para flutuações climáticas globais (IPCC, 2007). Os aerossóis podem afetar o clima através de absorção e dispersão da radiação solar (PENNER et al., 1994) e, por conseguinte, perturbar o balanço de radiação e contribuir para a forçante radioativa (ALPERT et al., 1998).

Aerossóis alteram o tamanho e a densidade das gotículas de nuvem modificando assim o albedo de nuvem, o tempo de vida de nuvem e a precipitação (RAMANATHAN et al., 2001). Aerossóis são uma das maiores fontes de incerteza nas avaliações e previsões sobre as alterações climáticas globais atuais (HANSEN et al., 2000). Aerossóis também influenciam a qualidade do ar e, por conseguinte, afetam a saúde humana (POPE LII et al., 2002), e reduzem a visibilidade de forma a afetar o tráfego.

Propriedades ópticas de aerossol, por exemplo, profundidade óptica de aerossóis (AOD) e expoente Ångström (EA), são fatores-chave para a investigação das alterações climáticas (KAUFMAN et al., 2002). Devido à sua alta variabilidade espacial-temporal, o monitoramento de aerossóis apresenta uma tarefa difícil, e esforços, assim, significativos são feitos para melhorar a caracterização de aerossóis usando medições in situ (HAYWOOD et al., 2003), como observações de sensoriamento remoto terrestre (HOLBEN et al., 2001) e observações de satélite (KING et al., 1999).

Em particular, a detecção remota por satélite, como MODIS torna-se uma maneira eficiente para monitorar propriedades ópticas de aerossóis (WANG et al., 2010). Os produtos de aerossol MODIS, fornecem a capacidade de monitorar as características espaço-temporais de aerossol mundial, obter as propriedades de aerossol, tanto sobre continentes (KAUFMAN et al., 1997), oceanos, utilizando sete canais espectrais $(0,47-2,1 \mathrm{~mm})$.

A região amazônica tem sofrido nas últimas décadas significativa mudança no padrão de uso do solo, através do intenso processo de ocupação humana (NOBRE et al., 1996). Estas alterações no uso do solo são responsáveis por emissões significativas de partículas de aerossóis para a atmosfera, através das queimadas, tanto de áreas de pastagem quanto de floresta primária (ARTAXO et al., 2001). Além das emissões de queimadas, a floresta amazônica, por sua localização tropical e seu intenso metabolismo, é uma importante fonte 
natural de gases traços, aerossóis e vapor de água para atmosfera global (ANDREAE; CRUTZEN, 1997). A intensa atividade convectiva leva estes constituintes atmosféricos a grandes altitudes, onde podem ser transportados de modo eficiente a longa distância (ANDREAE et al., 2001; FREITAS et al., 2005).

A região também é uma das principais fontes naturais de compostos orgânicos voláteis para a atmosfera global (GUENTHER et al., 1995), com significativas emissões de hidrocarbonetos como isopreno e terpeno, além de uma grande variedade de compostos oxigenados.

A ampla disponibilidade de radiação solar e a grande quantidade de vapor de água na atmosfera são características que favorecem uma alta reatividade química atmosférica na região tropical (ANDREAE; CRUTZEN, 1997). A floresta fornece, também, a maior parte das partículas que atuam como núcleos de condensação de nuvens ( $\mathrm{NCN})$, controlando efetivamente os mecanismos de formação de nuvens e precipitação (ARTAXO et al., 2002).

A composição química da atmosfera amazônica sofre grandes mudanças na época da seca, devido às emissões de gases traço e partículas de aerossóis provenientes de queimadas de pastagens e florestas, gerando importantes implicações em nível local, regional e global (ARTAXO et al., 2002). A cidade de Manaus encontra-se inserida na região amazônica, com aproximadamente 2.057.711 milhões de habitantes, cobrindo uma área de $11.401 .092 \mathrm{~km}^{2}$. O recente aumento de atividades agrícolas e de urbanização nessa área tem provocado impactos sobre aerossóis atmosféricos e na qualidade do ar.

Nesse sentido o objetivo desse trabalho foi realizar análise espectral da profundidade óptica de aerossóis (AOD 440nm) e expoente Ångström $\alpha$ (440-870) do sítio de Manaus_Embrapa por serem parâmetros básicos para descrição de aerossóis.

\section{MATERIAL E MÉTODOS}

O local escolhido para a realização da pesquisa foi um sítio com fotômetro solar da rede AERONET (Aerosol Robotic Rede) localizado em Manaus (Figura 1), sendo utilizado como base para coleta de dados nessa pesquisa. Sítio Manaus Embrapa localizado a $2.89^{\circ} \mathrm{S}, 59.96^{\circ} \mathrm{W}$, estando situado a 115 metros acima do nível do mar, em região extremamente populosa e industrializada. Instalado no topo de uma caixa de água com aproximadamente $115 \mathrm{~m}$.

Foram utilizados nesta pesquisa uma série temporal de dados de 4 anos de AOD(440nm), nível 1.5, confiáveis e disponibilizados após o tratamento.

\section{RESULTADOS}

3.1. Climatologia e Evolução Temporal AOD (440nm)

A alta resolução temporal dos dados AERONET permite estudar detalhadamente a evolução temporal de AOD (440nm). Na Figura 2 temos a variação de AOD (440nm).

A estatística geral para a série temporal de dados está representada na Tabela 1. A variabilidade sazonal de $\mathrm{AOD}(0 \mathrm{~nm})$ pode ser analisada pelas médias mensais apresentadas na Figura 3.

A variabilidade sazonal pode ser analisada pelas médias mensais de $\alpha(440-870 \mathrm{~nm})$ apresentadas na Figura 4 (também Tabela 1). A média mensal de água precipitável (AP) também foi avaliada, conforme variabilidade observada na Figura 5.

A evolução temporal de $\alpha(440-870 \mathrm{~nm})$ para o período de 2011-2014 é apresentado na Figura 6.

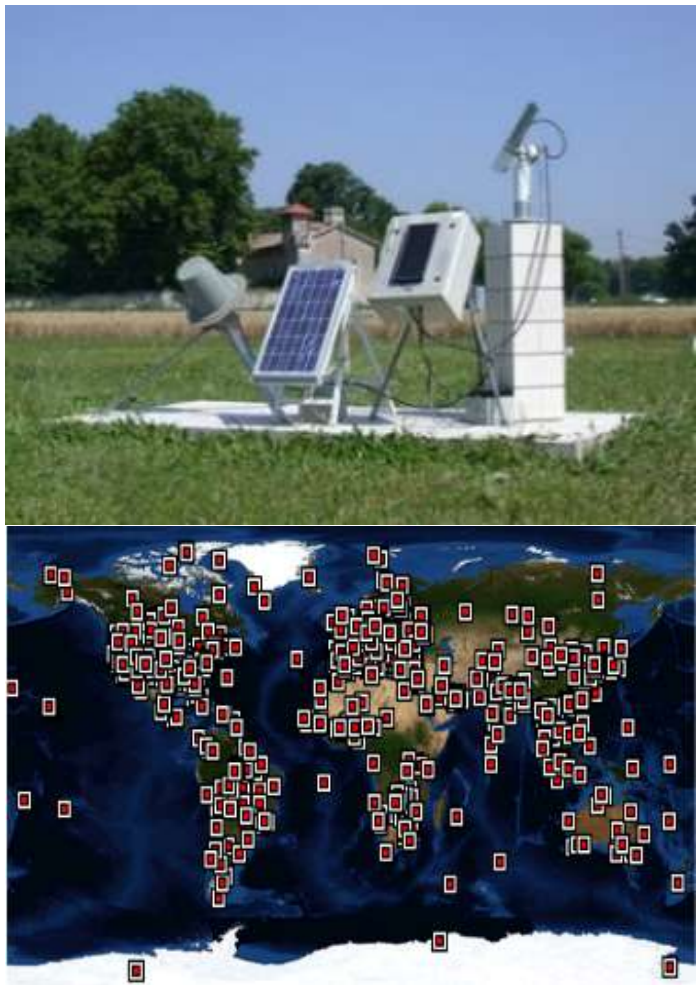

Figura 1. Fotômetros solares e localização do sítio AERONET. Figura 1. Solar photometer and location of AERONET sites.

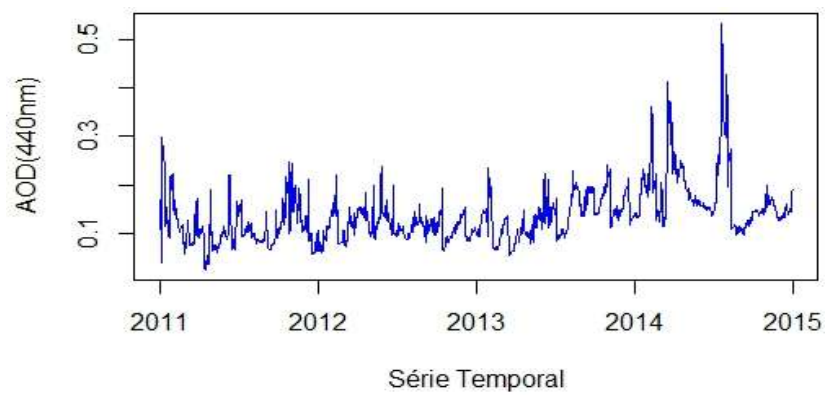

Figura 2. AOD(nm) de 2011 a 2014.

Figure 2. AOD (440nm) from 2011 to 2014.

Tabela 1. Estatística de AOD e Expoente Ångström em todos os comprimentos de onda.

Table 1. Statistics of AOD and Ångström expoent at all wavelengths.

\begin{tabular}{|c|c|c|c|c|c|}
\hline Variável & $\begin{array}{c}\text { AOD } \\
(1020)\end{array}$ & $\begin{array}{l}\text { AOD } \\
(870)\end{array}$ & $\begin{array}{l}\text { AOD } \\
(670)\end{array}$ & $\begin{array}{l}\text { AOD } \\
(440)\end{array}$ & $\begin{array}{c}\text { A } \\
(440-870)\end{array}$ \\
\hline Média & 0,078 & 0,095 & 0,126 & 0,221 & 1,279 \\
\hline Desvio Padrão & 0,170 & 0,183 & 0,233 & 0,403 & 0,394 \\
\hline Mínimo & 0,004 & 0,012 & 0,010 & 0,026 & 0,065 \\
\hline Máximo & 0,966 & 0,952 & 0,946 & 1,156 & 2,293 \\
\hline Mediana & 0,061 & 0,076 & 0,100 & 0,174 & 1,301 \\
\hline
\end{tabular}

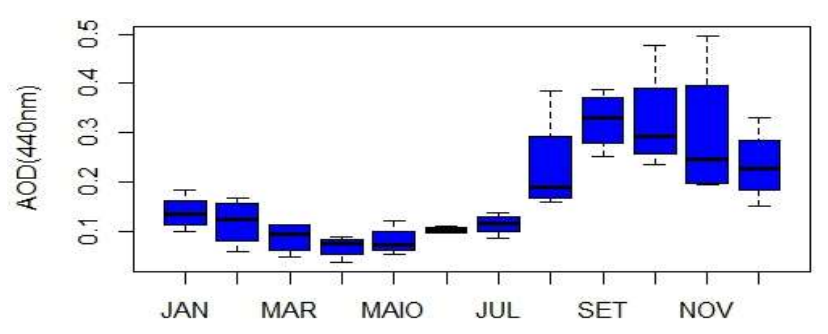

Figura 3. Média mensal de AOD(440nm) de 2011 a 2014.

Figure 3. Monthly average of AOD (440nm) for 2011-2014. 


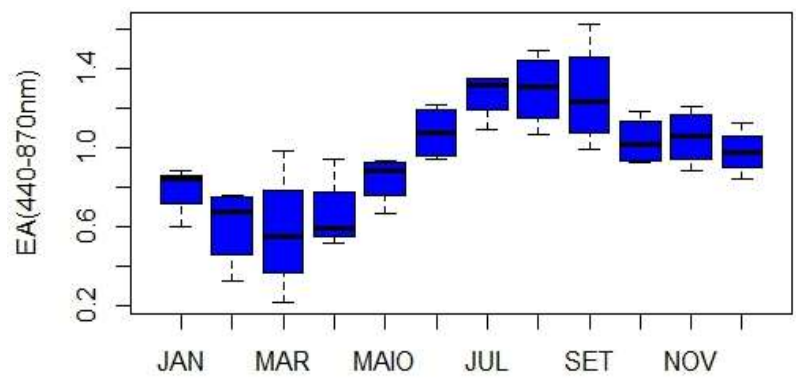

Figura 4. Média mensal do Expoente Ångström de 2011 a 2014. Figure 4. Monthly Average of Ångström Expoent from 2011 to 2014.

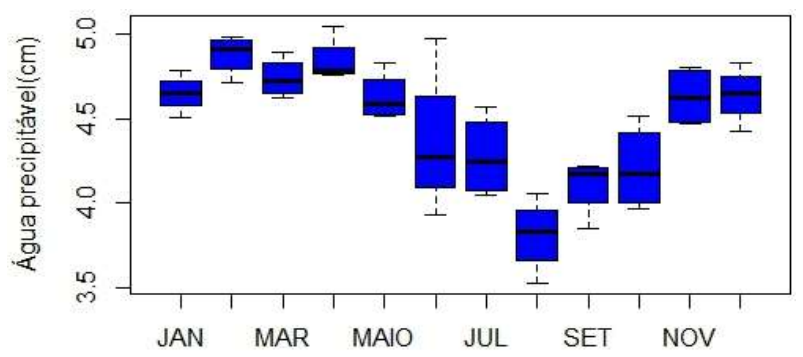

Figura 5. Média mensal de água precipitável de 2011 a 2014. Figure 5. Monthly average precipitable water from 2011-2014.

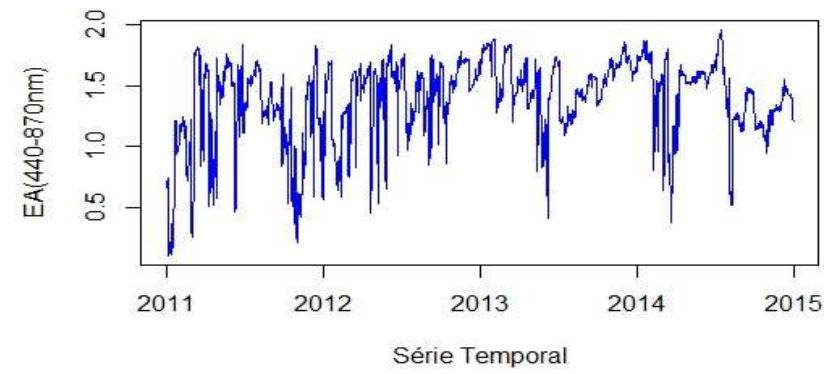

Figura 6. Gráfico de Evolução Temporal do Expoente Ångström de 2011 a 2014

Figure 6. Temporal Evolution Chart of the Ångström Exponent from 2011 to 2014.

Na Tabela 2 abaixo observa-se variação significativa ano a ano nas propriedades dos aerossóis. Variações do expoente Ångström também são observadas no período analisado.

Tabela 2. Média anual e desvio padrão de AOD(440nm), EA(440$870 \mathrm{~nm})$ e água precipitável.

Table 2. Annual mean and standard deviation (SD) of AOD (440nm), EA (440-870nm) and precipitated water (AP).

\begin{tabular}{ccccccc}
\hline Ano & $\begin{array}{c}\text { AOD } \\
(440)\end{array}$ & DP & $\begin{array}{c}\text { EA } \\
(440-870)\end{array}$ & DP & $\begin{array}{c}\text { AP } \\
(\mathrm{cm})\end{array}$ & DP \\
\hline 2011 & 0,154 & 0,092 & 1,002 & 0,288 & 4,444 & 0,457 \\
2012 & 0,214 & 0,149 & 0,965 & 0,38 & 4,501 & 0,269 \\
2013 & 0,143 & 0,08 & 0,839 & 0,205 & 4,459 & 0,293 \\
2014 & 0,196 & 0,115 & 1,013 & 0,296 & 4,488 & 0,444 \\
\hline
\end{tabular}

3.2. Climatologia e Evolução Temporal do Expoente Ångström

O gráfico de dispersão $\alpha(440-870 \mathrm{~nm})-\operatorname{AOD}(440 \mathrm{~nm})$ é uma ferramenta comum para classificar e caracterizar os tipos de aerossóis conforme TOLEDANO et al., (2007). Enquanto o $\operatorname{AOD}(440 \mathrm{~nm})$ dá informações sobre a quantidade de aerossóis, $\alpha(440-870 \mathrm{~nm})$ relaciona-se com o tamanho do aerossol (tipo), a análise conjunta de ambos os parâmetros torna possível uma melhor interpretação dos dados. Com análise espectral de aerossóis é possível a identificação dos principais tipos de aerossóis, bem como avaliar a frequência de cada tipo de aerossol presente (HOLBEN et al., 2001).

A análise do espectro de AOD $(440 \mathrm{~nm})$ versus $\alpha(440-870$ $\mathrm{nm})$, conforme Figura 7 abaixo, permite observar a variabilidade de AOD e EA na estação seca e chuvosa.

A análise do espectro de $\operatorname{AOD}(440 \mathrm{~nm})$ versus $\alpha(440$ $870 \mathrm{~nm}$ ), conforme Figura 8 abaixo, permite a identificação dos principais tipos de aerossóis presentes no sítio de Manaus_Embrapa.

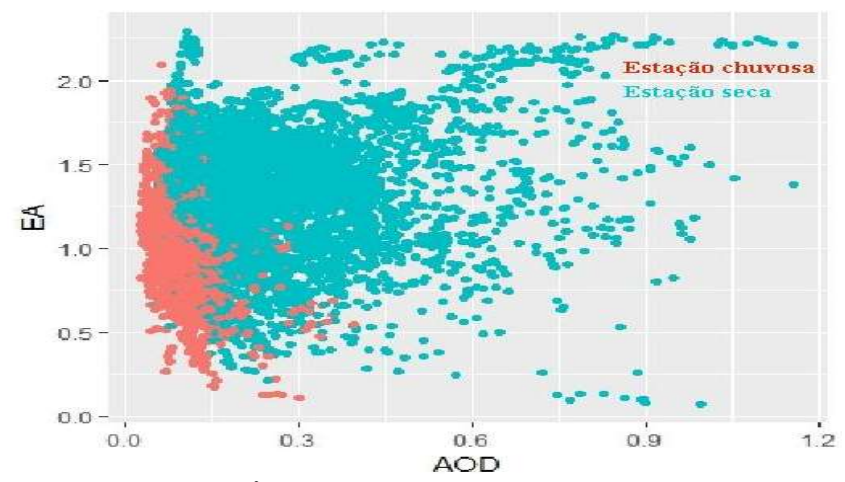

Figura 7. Expoente Ångström x AOD para estação seca e chuvosa de 2011 a 2014.

Figure 7. Ångström expoent x AOD for dry and rainy season 2011 to 2014.

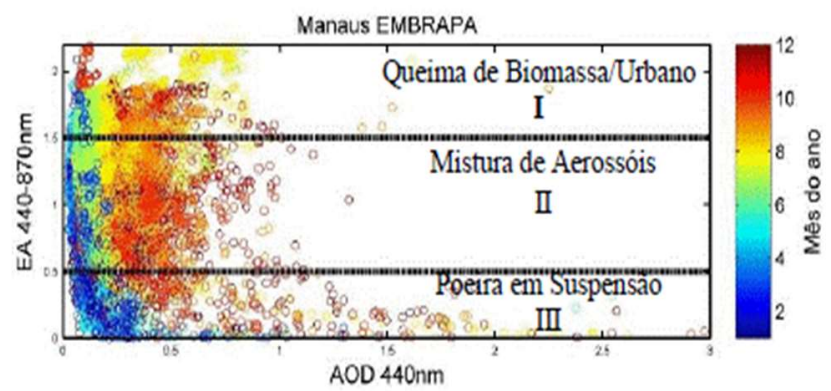

Figura 8. Gráfico de Expoente Ångström versus AOD(440nm) Figure 8. Expoent Ångström versus AOD (440nm) graph.

\section{DISCUSSÃO}

$\mathrm{Na}$ Figura 2 observa-se que uma série de episódios com AOD $(440 \mathrm{~nm})$ no intervalo de 0,026-1,156 ocorre nesse sítio. Observa-se que há uma variabilidade ano-a-ano de AOD $(440 \mathrm{~nm})$ para a série de dados analisada. A AOD $(440 \mathrm{~nm})$ para esse sítio, mostra uma tendência crescente em quase todos os anos. A principal característica de $\operatorname{AOD}(440 \mathrm{~nm})$ é sua alta variabilidade (TOLEDANO et al., 2007).

A AOD $(440 \mathrm{~nm})$ observada variaram aproximadamente em toda a estação para a série temporal de dados considerada, de 0,02 a 0,99 para o ano de 2011 , de aproximadamente 0,03 a 1,15 para o ano de 2012 , de 0,02 a 0,88 para o ano de 2013 e variações de 0,02 a 1,15 para o ano de 2014. O aumento da variabilidade de AOD(440nm) observada pode estar estreitamente relacionada com mudanças nos padrões climáticos locais.

Os aerossóis oriundos de queima de biomassa são os principais responsáveis pela ocorrência desses episódios. A 
variabilidade observada é maior para comprimentos de onda menores.

Observa-se da Tabela 1 que a média de $\operatorname{AOD}(440 \mathrm{~nm})$ é 0,22, com desvio padrão de $\operatorname{AOD}(440 \mathrm{~nm})$ de 0,40 , representando variações típicas para essas regiões, com máximo de 1,15 . A média de $\operatorname{AOD}(440 \mathrm{~nm})$ diminui para comprimentos de onda maiores, tendo média de AOD(1020nm) 0,07. Observa-se média de 1,2 para $\alpha(440-$ $870 \mathrm{~nm})$, com desvio padrão de 0,39 e apresentando máximo de 2,29 . Os valores máximos de $\mathrm{AOD}(440 \mathrm{~nm})$ podem indicar alta variabilidade nesse sítio.

A média mensal de $\operatorname{AOD}(440 \mathrm{~nm})$ foi utilizada para investigar a sazonalidade desses dados, sendo que os máximos de AOD(440nm) durante a estação seca, conforme Figura 3, foram observados nos meses de agosto a dezembro, tendo um decréscimo após a estação seca. As diferenças de valores mensais de $\alpha(440-870 \mathrm{~nm})$ ao longo dos anos são crescentes, considerando que os maiores valores são observados entre os meses de julho, agosto e setembro, conforme Figura 4.

Observa-se menores valores de água precipitável, Figura 5, na atmosfera desse sítio nos meses de agosto a outubro (estação seca), com crescente aumento após a estação seca.

Na Figura 6 observa-se alta variabilidade, com valores na faixa de 0,06 a 2,29 ao longo de toda a série de dados. Não se observa valores negativos ao longo da série, que se relacionam com dias nublados e provavelmente com contaminação de nuvem para ambos os sítios. Pelo contrário, durante a estação seca os valores de $\operatorname{AOD}(440 \mathrm{~nm})$ mais altos, acima de 1,5, em média, resultam em valores recuperados mais precisos, permitindo a identificação e monitoramento de eventos com alto $\operatorname{AOD}(440 \mathrm{~nm})$ e baixo $\alpha(440-870 \mathrm{~nm})$ e eventos relacionados com alto $\mathrm{AOD}(440 \mathrm{~nm})$ e alto $\alpha(440-870 \mathrm{~nm})$.

A AOD(440nm) da série de dados, conforme Tabela 2, varia de 0,03 a 0,71 numa base diária. A média mensal varia de 0,03 a 0,94. Estas altas médias mensais de $\operatorname{AOD}(440 \mathrm{~nm})$ (acima de 0,2) mostram o nível de poluição na atmosfera consistentemente durante um período prolongado.

Em escala anual, o valor mínimo de $\operatorname{AOD}(440 \mathrm{~nm})$ de 0,143 ocorre em 2013 quando o teor de água (PW) da atmosfera não estava no seu pico em comparação com qualquer outro ano. Ao investigar as causas dos AODs mais altos e mais baixos nos anos de 2012 e 2013, verificou-se que a deposição húmida, descrita como o processo mais eficiente de remoção de aerossóis foi máxima (maior AP) em 2012 e menor (menor AP) durante 2013. Como resultado disto, apenas uma menor concentração de partículas finas está presente na atmosfera em comparação com a concentração de partículas maiores (menor $\alpha$ ) encontrada em 2013, dando origem ao $\mathrm{AOD}(440 \mathrm{~nm})$ máximo de 0,214 em 2012.

A grande variabilidade $( \pm 0,14)$ nos valores de $\operatorname{AOD}(440 \mathrm{~nm})$ observados durante o ano indica um grande aumento na concentração do modo grosseiro na atmosfera. No entanto, a variabilidade foi baixa em 2013, resultante de uma diminuição significativa nas concentrações de aerossóis, possivelmente devido a regulamentações governamentais no controle da poluição por aerossóis.

A maioria dos valores de $\alpha(440-870 \mathrm{~nm})$ para os anos de 2011 e 2014 apresentam valores maiores que 1, indicando a predominância de partículas de moda fina. Nos anos de 2012 e 2013 esses valores são inferiores a 1, indicando predominância de moda grossa nessa região. No entanto, os valores de $\alpha(440-870 \mathrm{~nm})$ apresentam um padrão de flutuação quase semelhante em cada estação, embora alguns valores altos sejam visíveis durante o período de estação seca em comparação com estação chuvosa, indicando a dominância de partículas finas e grossas em cada estação. Isto significa que, embora a presença de chuva diminua as concentrações sazonalmente, a área de estudo tem sido dominada por aerossóis maiores ano após ano, que é ou aerossol oriundo de queima de biomassa e/ou mistura de material particulado.

Os valores de $\alpha(440-870 \mathrm{~nm})$, Figura 6 , variam de 0,2 a 1,35 em uma base mensal, enquanto 0,83 a 1,01 em uma escala anual. O teor de água na atmosfera (água precipitável) como mostrado na Figura 5 acima ocorre mais frequentemente em valores mais baixos $(1,36-3,63 \mathrm{~cm})$ durante a estação seca que corresponde aos meses de agosto a outubro, onde as concentrações de aerossóis são altas.

Foram feitos gráficos de dispersão de $\alpha(440-870 \mathrm{~nm})$ contra AOD (440nm), para estação seca e chuvosa, sendo apresentados na Figura 7 para o sítio de Manaus_Embrapa. As escalas de cores nos gráficos indicam o número de medidas realizadas em diferentes estações.

Para os valores observados na estação seca de $\alpha(440$ $870 \mathrm{~nm})$ superiores a 1,5 e $\operatorname{AOD}(440 \mathrm{~nm})(<1.2)$, tem-se aerossóis oriundos de queima de biomassa e/ou aerossóis urbanos e/ou áreas industriais, sendo que tais eventos são mais intensos nesse sítio.

Quando AOD(440nm) é baixo temos valores de $\alpha(440-$ $870 \mathrm{~nm})$ sobre todo alcance, porém, principalmente concentrado em torno de uma região de 0,065-2,293. Assim, podemos observar baixos valores de $\operatorname{AOD}(440 \mathrm{~nm})$ na estação chuvosa. $\mathrm{Na}$ estação seca observamos um crescimento progressivo dos valores de $\operatorname{AOD}(440 \mathrm{~nm})$ nesse sítio. Para os maiores valores de $\operatorname{AOD}(440 \mathrm{~nm})$, a situação é mais definida; $\alpha(440-870 \mathrm{~nm})$ está compreendido abaixo ou acima de 1,30. A análise desses ambientes revela a presença de diferentes tipos de aerossóis.

Os produtos de $\operatorname{AOD}(440 \mathrm{~nm})$ e $\alpha(440-870 \mathrm{~nm})$ podem prover informações sobre características de absorção e extinção e distribuição de tamanho de aerossóis na atmosfera. Em geral, altos valores de $\operatorname{AOD}(440 \mathrm{~nm})$ estão associados a alta extinção (absorção) da radiação, e pelas mesmas razões, altos valores de $\alpha(440-870 \mathrm{~nm})$ estão associados a distribuição de tamanho de aerossóis de moda fina. Essas duas características são consideradas a assinatura de aerossóis de queima de biomassa.

Há uma vasta gama de valores $\alpha(440-870 \mathrm{~nm})$ inferiores a 0,5 com baixo AOD $440 \mathrm{~nm}(<0.4)$ observados na estação chuvosa, podendo ser caracterizados como poeira em suspensão, conforme Figura 8.

Ainda na estação chuvosa, observa-se valores de $\alpha(440$ $870 \mathrm{~nm}$ ) compreendidos entre 0,5 e 1,5 referente a mistura de aerossóis característicos desses sítios, sendo que os valores crescentes de $\alpha(440-870 \mathrm{~nm})$ com o aumento significativo da $\operatorname{AOD}(440 \mathrm{~nm})$ indicam contribuição de finas partículas na coluna atmosférica (KASKAOUTIS et al., 2008). Uma relação semelhante entre concentração de partículas (através de AOD) e tamanho de partícula (por meio do expoente Ångström) foi observada para uma variedade de aerossóis de moda fina (REMER et al., 1998).

\section{CONCLUSÕES}

Registros (2011-2014) de AOD(440nm) e Expoente Ångström $\alpha(440-870 \mathrm{~nm})$, de dados AERONET, foram usados para estudar as variações sazonais de aerossóis, características e tipos, sob diferentes condições atmosféricas em sítio 
localizado em área de Floresta Tropical, sítio de Manaus Embrapa. Este estudo mostrou que variações sazonais nesse sítio resultam de diferentes concentrações de aerossóis, características e tipos dominantes. Baixos valores de $\operatorname{AOD}(440 \mathrm{~nm})$ e dependência do comprimento de onda são observados na estação chuvosa, tendo valores elevados de $\operatorname{AOD}(440 \mathrm{~nm})$ na estação seca.

\section{AGRADECIMENTOS}

Agradecemos ao Dr. Brent Holben e Dr. Paulo Artaxo pelo esforço no estabelecimento e manutenção do site aeronet.gsfc.nasa.gov.

\section{REFERÊNCIAS}

ALPERT, P.; KAUFMAN, Y. J.; SHAY-EL, Y.; TANRE, D.; DA SILVA, A.; SCHUBERT, S.; JOSEPH, J. H. Quantification of dust-forced heating of the lower atmosphere. Nature, p. 367-370, 1998. DOI: https://doi.org/10.1038/26456

ANDREAE, M. O.; ARTAXO, P.; FISCHER, H.; FREITAS, S. R.; GRÉGOIRE, J.-M.; HANSEL, A.; HOOR, P.; KORMANN, R.; KREJCI, R.; LANGE, L.; LELIEVELD, J.; LINDINGER, W.; LONGO, K.; PETERS, W.; DE REUS, M.; SCHEEREN, B.; SILVA DIAS, M. A. F.; STRÖM, J.; VAN VELTHOVEN, P. F. J.; WILLIAMS, J. Transport of biomass burning smoke to the upper troposphere by deep convection in the equatorial region. Geophysical Research Letters, v. 28, n. 6, p. 951-954, 2001. DOI: https://doi.org/10.1029/2000GL012391

ANDREAE, M. O.; CRUTZEN, P. J. Atmospheric Aerosols: biogeochemical sources and role in atmospheric chemistry. Science, v. 276, n. 5315, p. 1052-1058, 1997. DOI: https://doi.org/10.1126/science.276.5315.1052

ARTAXO, P. The atmospheric component of biogeochemical cycles in the Amazon basin. The biogeochemistry of the Amazon Basin, p. 42-52, 2001.

ARTAXO, P.; MARTINS, J. V.; YAMASOE, M. A.; PROCOPIO, A. S.; PAULIQUEVIS, T. M.; ANDREAE, M. O.; GUYON, P.; GATTI, L. V.; CORDOVA, A. M. Physical and chemical properties os aerosols in the wet and dry season in Rondônia, Amazonia. Journal of Geophysical Research, v. 107(D20), p. 8081-8095, 2002. DOI: https://doi.org/10.1029/2001JD000666

FREITAS, S. R.; LONGO, K. M.; SILVA DIAS, M. A. F.; SILVA DIAS, P. L.; CHATFIELD, R.; PRINS, E.; ARTAXO, P.; GRELL, G. A.; RECUERO, F. S. Monitoring the transport of biomass burning emissions in South America. Environmental Fluid Mechanics, v. 5, n. $1-2, \quad$ p. $135-167, \quad 2005$. DOI http://dx.doi.org/10.5094/APR.2011.031.

GUENTHER, A.; HEWITT, C. N.; ERICKSON, D.; FALL, R.; GERON, C.; GRAEDEL, T.; HARLEY, P.; KLINGER, L.; LERDAU, M.; MCKAY, W. A.; PIERCE, T.; SCHOLES, B.; STEINBRECHER, R.; TALLAMRAJU, R.; TAYLOR, J.; ZIMMERMAN, P. A. A global-model of natural volatile organic-compound emissions. Journal of Geophysical Research Atmosphere, v. 100, n. D5, p. 8873-8892, 1995. DOI: https://doi.org/10.1029/94JD02950

HANSEN, J.; SATO, M.; RUEDY, R.; LACIS, A.; OINAS, $\mathrm{V}$. Global warming in the twenty-first centry: ann alternative scenario. Proceedings of the National
Academy of Sciences of the United States of America, v. $97, \quad$ n. $18, \quad$ p. $9875-9880,2000$. DOI: https://doi.org/10.1073/pnas.170278997

HAYWOOD, J.; FRANCIS, P.; DUBOVIK, O.; GLEW, M.; HOLBEN, B. Comparision of aerosol size distributions, radiative properties, and optical depths determined by aircraft observations and Sun photometers during SAFARI 2000. Journal of Geophysical Research, v. 108, n. D13, p. 7.1-7.12, 2003 . DOI: https://doi.org/10.1029/2002JD002250

HOLBEN, B. N.; TANRE, D.; SMIRNOV, A.; ECK, T. F.; SLUTSKER, I.; ABUHASSAN, N.; NEWCOMB, W. W.; SCHAFER, J. S.; CHATENET, B.; LAVENU, F.; KAUFMAN, Y. J.; VANDE CASTLE, J.; SETZER, A.; MARKHAM, B.; CLARK, D.; FROUIN, R.; HALTHORE, R.; KARNELI, A.; O'NEILL, N. T.; PIETRAS, C.; PINKER, R. T.; VOSS, K.; ZIBORDI, G. An emerging ground-based aerosol climatology: aerosol optical depth from AERONET. Journal of Geophysical Research, v. 12, n. D11, p. 12067-12097, 2001. DOI: https://doi.org/10.1029/2001JD900014

IPCC. Cambridge Univesity Press. New York, p. 131-216. 2007.

KASKAOUTIS, D. G.; KAMBEZIDIS, H. D.; NASTOS, P. T.; KOSMOPOULOS, P. G. Study on an intense dust storm over Greece. Atmospheric Environment, v. 42, p. 6884-6896, 2008. DOI: http://dx.doi.org/10.1016/j.atmosenv.2008.05.017.

KAUFMAN, Y. J.; TANRE, D.; BOUCHER, O. A satellite view of aerosols in the climate system. Nature, n. 419, p. 215-223, $2002 . \quad$ DOI: https://doi.org/10.1038/nature01091

KAUFMAN, Y. J.; TANRE, D.; REMER, L. A.; VERMOTE, E. F.; CHU, A.; HOLBEN, B. N. Operational remote sensig of tropospheric aerosol over land from EOS moderate resolution imaging spectroradiometer. Journal of Geophysical Research, v. 102, n. 17, p. 17051-17067, 1997. DOI: https://doi.org/10.1029/96JD03988

KING, M. D.; KAUFMAN, Y. J.; TANRE, D.; NAKAJIMA, T. Remote sensing of tropospheric aerosols from space: paste, presente, and future. Bulletin of the American Meteorological Society, v. 80, p. 2229-2259, 1999. DOI: https://doi.org/10.1175/15200477(1999)080<2229:RSOTAF $>2.0$. CO 2

NOBRE, C. A.; LUIZÃO, F.; VICTORIA, R. L.; SILVA DIAS, P. L.; SILVA DIAS, M. A. F.; ARTAXO, P. The Large Scale Biosphere Atmosphere Experiment in Amazonia (LBA), Cachoeira Paulista, SP. Brazil, 1996.

PENNER, J. E.; CHARLSON, R. J.; LAULAINEM, N. S.; LEIFER, R.; NOVAKOV, T.; OGREN, J.; RADKE, L. F.; SCHWARTZ, S. E.; TRAVIS, L. Quantifying and minimizing uncetainty of climate forcing by anthropenic aerosols. Bulletin of the American Meteorological Society, p. 375-400, 1994. DOI: https://doi.org/10.1175/1520 0477(1994)075<0375:QAMUOC $>2.0 . C O ; 2$

POPE LII, C. A.; BURNET, R. T.; THUN, M. J.; CALLE, E. E.; KREWSKI, D.; ITO, K.; THURSTON, G. D. Lung cancer, cardiopulmonar mortality, and long-term exposure to fine particular air pollution. JAMA, v. 287, n. 9, p. 1132-1141, 2002 
RAMANATHAN, V.; CRUTZEN, P. J.; KIEHL, J. T.; ROSENFELD, D. Aerosols, climate, and the hydrological cycle. Science, v. 249, n. 5549, p. 2119-2124, 2001. DOI: https://doi.org/10.1126/science.1064034

REMER, L. A.; KAUFMAN, Y.; HOLBEN, B. N.; THOMPSON, A. M.; MCNAMARA, D. P. Biomass burning aerossol size distribution and modeled optical properties. Journal of Geophysical Research, v. 103, n. D24, p. 31879-31891, 1998. DOI: https://doi.org/10.1029/98JD00271

TOLEDANO, C.; CACHORRO, V. E.; BERJON, A.; DE FRUTOS, A. M.; SORRIBAS, M.; DE LA MORENA, B. A.; GOLOUB, P. Aerosol optical depth and Ångström expoent climatology at el arenosillo AERONET Site
(Huelva, Spain). Quartely Journal of the Royal Meteorological Society, v. 133, p. 795-807, 2007. DOI: https://doi.org/10.1002/qj.54

WANG, L. L.; WANG, Y. S.; XIN, J. Y.; LI, Z. Q.; WANG, $\mathrm{X}$. Y. Assessment and comparison of three years of Terra and Aqua MODIS aerosol optical depth retrieval (COO5) in Chinese terrestrial regions. Atmospheric Research, v. 97 , n. 1-2, p. 229-240, 2010. DOI: https://doi.org/10.1016/j.atmosres.2010.04.004 